\title{
The Complexity of König Subgraph Problems and Above-Guarantee Vertex Cover.*
}

\author{
Sounaka Mishra ${ }^{2}$, Venkatesh Raman ${ }^{1}$, Saket Saurabh ${ }^{1}$, Somnath Sikdar ${ }^{3}$ and C. R. \\ Subramanian $^{1}$ \\ 1 The Institute of Mathematical Sciences, \\ Chennai, India. \\ \{vraman|saket|crs\}@imsc.res.in \\ 2 The Indian Institute of Technology, \\ Department of Mathematics, Chennai, India. \\ sounak@iitm.ac.in \\ 3 Department of Computer Science, \\ RWTH Aachen University, 52056 Aachen, Germany \\ sikdar@cs.rwth-aachen.de
}

\begin{abstract}
A graph is König-Egerváry if the size of a minimum vertex cover equals that of a maximum matching in the graph. These graphs have been studied extensively from a graph-theoretic point of view. In this paper, we introduce and study the algorithmic complexity of finding KönigEgerváry subgraphs of a given graph. In particular, given a graph $G$ and a nonnegative integer $k$, we are interested in the following questions:

1. does there exist a set of $k$ vertices (edges) whose deletion makes the graph König-Egerváry?

2. does there exist a set of $k$ vertices (edges) that induce a König-Egerváry subgraph?

We show that these problems are NP-complete and study their complexity from the points of view of approximation and parameterized complexity. Towards this end, we first study the algorithmic complexity of Above Guarantee Vertex Cover, where one is interested in minimizing the additional number of vertices needed beyond the maximum matching size for the vertex cover. Further, while studying the parameterized complexity of the problem of deleting $k$ vertices to obtain a König-Egerváry graph, we show a number of interesting structural results on matchings and vertex covers which could be useful in other contexts.
\end{abstract}

\section{Introduction}

The classical notions of matchings and vertex covers have been at the center of serious study for several decades in the area of Combinatorial Optimization [21]. In 1931, König and Egerváry independently proved a result of fundamental importance: in a bipartite graph the size of a maximum matching equals that of a minimum vertex cover [21]. This led to a polynomial-time algorithm for finding a minimum vertex cover in bipartite graphs. In fact, a maximum matching can be used to obtain a 2-approximation algorithm for the Minimum VerTex Cover problem in general graphs, which is still the best-known constant-factor approximation algorithm for this problem [17]. Interestingly, this min-max relationship holds for a larger class of graphs known as König-Egerváry graphs and it includes bipartite graphs as a proper subclass. König-Egerváry graphs will henceforth be called König graphs.

König graphs have been studied for a fairly long time from a structural point of view $[2,6,19$, 20,32]. Both Deming [6] and Sterboul [32] gave independent characterizations of König graphs and showed that König graphs can be recognized in polynomial time. Lovász [20] used the theory

\footnotetext{
* Preliminary versions of this paper appeared in the proceedings of the 18th and 19th International Symposium on Algorithms and Computation (ISAAC 2007 and ISAAC 2008).
} 
of matching-covered graphs to give an excluded-subgraph characterization of König graphs that contain a perfect matching. Korach et al. [19] generalized this and gave an excluded-subgraph characterization for the class of all König graphs.

A natural optimization problem associated with a graph class $\mathcal{G}$ is the following: given a graph $G$, what is the minimum number of vertices to be deleted from $G$ to obtain a graph in $\mathcal{G}$ ? For example, when $\mathcal{G}$ is the class of empty graphs, forests or bipartite graphs, the corresponding problems are Vertex Cover, Feedback Vertex Set and Odd Cycle Transversal, respectively. We call the vertex-deletion problem corresponding to the class of König graphs the KÖNig Vertex Deletion problem. A set of vertices whose deletion makes a given graph König is called a König vertex deletion set. In the parameterized setting, the parameter for vertex-deletion problems is the solution size, that is, the number of vertices to be deleted so that the resulting graph belongs to the given graph class.

In this paper, we define various problems related to finding König-Egerváry subgraphs and study their algorithmic complexity from the points of view of parameterized complexity and approximation algorithms. More precisely the problems that we study in this paper are:

1. König Vertex (Edge) Deletion. Given a graph $G$ and a nonnegative integer $k$, do there exist at most $k$ vertices (respectively, edges) whose deletion results in a König subgraph?

2. Vertex (Edge) Induced König Subgraph. Given a graph $G$ and a nonnegative integer $k$, do there exist at least $k$ vertices (respectively, edges) which induce ${ }^{4}$ a König subgraph?

The König Vertex Deletion and Vertex Induced König Subgraph problems (and similarly, König Edge Deletion and Edge Induced König Subgraph) are equivalent from the point of view of NP-completeness but differ in their approximability and parameterized complexity.

The main technical contribution of this paper is in showing that the KöNIG VERTEX DeLETION problem is fixed-parameter tractable. To do this, we first establish interesting structural connections between minimum vertex covers, maximum matchings and minimum König vertex deletion sets. Using these, we show that KÖNIG VERTEX DELETION is fixed-parameter tractable when parameterized by the solution size. Note that König graphs are not hereditary, that is, not closed under taking induced subgraphs. For instance, a 3-cycle is not König but attaching an edge to one of the vertices of the 3-cycle results in a König graph. In fact, König VERTEX DELETION is one of the few vertex-deletion problems associated with a non-hereditary graph class whose parameterized complexity has been studied. Another such example can be found in $[25]$.

One motivation for studying König subgraph problems is that the versions of König subgraph problems when the resulting graph we look for is bipartite (i.e. replace König in the above problem definitions by bipartite) are well studied in the area of approximation algorithms and parameterized complexity [14,30,31]. König subgraph problems are natural generalizations of bipartite subgraph problems but have not been studied algorithmically. We believe that this can trigger explorations of other questions in König graphs.

Another motivation for studying König subgraph problems is that KöNIG VERTEX DeLETION is closely related to a problem known as Above Guarantee Vertex Cover. The latter is a variant of the classical VERTEX COVER problem and is defined as follows: given a

\footnotetext{
${ }^{4}$ If $E^{\prime} \subseteq E$ is a set of edges, then the graph $G\left[E^{\prime}\right]$ induced by $E^{\prime}$ is one whose vertex-set is the set of endpoints of $E^{\prime}$ and whose edge-set is the set $E^{\prime}$.
} 
graph $G=(V, E)$ and a nonnegative integer $k$, decide whether $G$ has a vertex cover of size at most $\mu(G)+k$, where $\mu(G)$ is the size of a maximum matching of $G$. This problem was first introduced by Subramanian in [33]. Note that the parameter $k$ is the size of the solution beyond the matching size. This parameterization is more meaningful than the standard parameterized version of VERTEX COVER where the parameter is the size of the entire solution, since in graphs with a perfect matching the solution is at least half the number of vertices in the graph. A brute-

force algorithm for VERTEX COVER that checks all vertex-subsets is a fixed-parameter algorithm in this case. The parameterized complexity of Above Guarantee Vertex Cover was open for quite some time and is now known to be fixed-parameter tractable due to the results in [29] and in this paper.

This paper is organized as follows. In Section 2 we give a brief outline of parameterized complexity, describe our notation and state some known results about König graphs. In Section 3 we show that Above Guarantee Vertex Cover is fixed-parameter tractable by reducing it to a (fixed-parameter tractable) problem called Min 2-CnF SAT DeL (Theorem 1). We show how to use an $O(\log n \log \log n)$-approximation algorithm for Min 2-CNF SAT DeL to obtain an approximation algorithm for Above Guarantee Vertex Cover with the same asymptotic performance ratio (Theorem 2). In addition, we show that neither of these problems admit constant-factor approximation algorithms unless the Unique Games ConjeCture (UGC) [16] is false (Theorem 3, Corollary 1).

Section 4 is the main technical section of the paper. Here we study the parameterized complexity and approximability of König Vertex Deletion. We show that for graphs with a perfect matching, König Vertex Deletion is fixed-parameter equivalent to Above GuarANTEE Vertex COVER. This immediately establishes the fixed-parameter tractability of KÖNIG Vertex Deletion on graphs with a perfect matching. For general graphs, we first establish some structural connections between minimum vertex covers, maximum matchings and minimum König vertex deletion sets. Using these, we exhibit a reduction from KöNIG VERTEX DELETION to Above Guarantee Vertex Cover, which then establishes the fixed-parameter tractability of the former (Theorem 4). The approximability of KÖNIG VERTEX DELETION is similar to that of Above Guarantee Vertex Cover. It does not admit a constant-factor approximation algorithm unless UGC is false and can be approximated to within a factor of $O(\log n \log \log n)$ (Theorem 5, Corollary 10).

In Section 5 we study vertex and edge versions of the INDUCED KÖNIG SubGRAPH problem. We show that Vertex Induced KÖNIG SUBGRAPH is unlikely to be fixed-parameter tractable (that it is $W[1]$-hard) and that it is inapproximable to within a factor of $O\left(n^{1-\epsilon}\right)$, for any $\epsilon>$ 0 (Theorem 6, Corollary 12). We also show that König EdGe Induced Subgraph is NPcomplete (Theorem 7), has a constant-factor approximation algorithm (Theorem 8) and is fixedparameter tractable (Theorem 9). Similar to its vertex counterpart, KÖNIG EdGE DELETION does not admit constant-factor approximation algorithms unless UGC is false (Corollary 13). We conclude in Section 6 with some open problems among which is the parameterized complexity of KÖNIG EDGE DeLETION.

\section{Preliminaries}

In this section we summarize the necessary concepts concerning parameterized complexity, fix our notation and describe some well-known properties of König graphs. 


\subsection{Parameterized Complexity}

A parameterized problem is a subset of $\Sigma^{*} \times \mathbb{Z}^{\geq 0}$, where $\Sigma$ is a finite alphabet and $\mathbb{Z}^{\geq 0}$ is the set of nonnegative numbers. An instance of a parameterized problem is therefore a pair $(I, k)$, where $k$ is the parameter. In the framework of parameterized complexity, the running time of an algorithm is viewed as a function of two quantities: the size of the problem instance and the parameter. A parameterized problem is said to be fixed-parameter tractable (FPT) if there exists a deterministic algorithm that takes as input $(I, k)$ and decides whether it is a YES or NO-instance in time $O\left(f(k) \cdot|I|^{O(1)}\right)$, where $f$ is a function depending only on $k$. The class FPT consists of all fixed-parameter tractable problems.

A parameterized problem $\pi_{1}$ is fixed-parameter reducible to a parameterized problem $\pi_{2}$ if there exist functions $f, g: \mathbb{Z}^{\geq 0} \rightarrow \mathbb{Z}^{\geq 0}, \Phi: \Sigma^{*} \times \mathbb{Z}^{\geq 0} \rightarrow \Sigma^{*}$ and a polynomial $p(\cdot)$ such that for any instance $(I, k)$ of $\pi_{1},(\Phi(I, k), g(k))$ is an instance of $\pi_{2}$ computable in time $f(k) \cdot p(|I|)$ and $(I, k) \in \pi_{1}$ if and only if $(\Phi(I, k), g(k)) \in \pi_{2}$. Two parameterized problems are fixed-parameter equivalent if they are fixed-parameter reducible to each other. The basic complexity class for fixed-parameter intractability is $W[1]$ as there is strong evidence that $W[1]$-hard problems are not fixed-parameter tractable. To show that a problem is $W[1]$-hard, one can exhibit a fixedparameter reduction from a known $W[1]$-hard problem to the problem at hand. For more on parameterized complexity see $[8,9,27]$.

\subsection{Notation}

Given a graph $G$, we use $\mu(G), \beta(G)$ and $\kappa(G)$ to denote, respectively, the size of a maximum matching, a minimum vertex cover and a minimum König vertex deletion set of $G$. We sometimes use $\tau(G)$ to denote the difference $\beta(G)-\mu(G)$. When the graph being referred to is clear from the context, we simply use $\mu, \beta, \kappa$ and $\tau$. Given a graph $G=(V, E)$ and two disjoint vertex subsets $V_{1}, V_{2}$ of $V$, we let $\left(V_{1}, V_{2}\right)$ denote the bipartite graph with vertex set $V_{1} \cup V_{2}$ and edge set $\left\{\{u, v\}:\{u, v\} \in E\right.$ and $\left.u \in V_{1}, v \in V_{2}\right\}$. If $B$ is a bipartite graph with vertex partition $L \uplus R$ then we let $\mu(L, R)$ denote the size of the maximum matching of $B$. If $M$ is a matching and $\{u, v\} \in M$ then we say that $u$ is the partner of $v$ in $M$. If the matching being referred to is clear from the context we simply say $u$ is a partner of $v$. The vertices of $G$ that are the endpoints of edges in the matching $M$ are said to be saturated by $M$; all other vertices are unsaturated by $M$.

\subsection{Properties of König Graphs}

A graph $G=(V, E)$ is said to be König if $\beta(G)=\mu(G)$. The following lemma follows directly from the definition of König graphs.

Lemma 1. [6,32] A graph $G=(V, E)$ is König if and only if for every bipartition of $V$ into $V_{1} \uplus V_{2}$, with $V_{1}$ a minimum vertex cover of $G$, there exists a matching across the cut $\left(V_{1}, V_{2}\right)$ saturating every vertex of $V_{1}$.

In order to show that a graph is König it is actually sufficient to demonstrate the existence of just one bipartition of $V$ into $V_{1} \uplus V_{2}$, with $V_{1}$ a vertex cover of $G$ such that there exists a matching across the cut $\left(V_{1}, V_{2}\right)$ saturating every vertex of $V_{1}$. 
Lemma 2. A graph $G=(V, E)$ is König if and only if there exists a bipartition of $V$ into $V_{1} \uplus V_{2}$, with $V_{1}$ a vertex cover of $G$ such that there exists a matching across the cut $\left(V_{1}, V_{2}\right)$ saturating every vertex of $V_{1}$.

Proof. If $G$ is König then, by Lemma 1 , there exists a bipartition $V_{1} \uplus V_{2}$, with $V_{1}$ a minimum vertex cover of $G$, such that there exists a matching across the cut $\left(V_{1}, V_{2}\right)$ saturating every vertex of $V_{1}$. Conversely suppose that the vertex set of $G$ can be partitioned as $V_{1} \uplus V_{2}$ such that $V_{1}$ is a vertex cover and there exists a matching $M$ across the cut $\left(V_{1}, V_{2}\right)$ saturating every vertex of $V_{1}$. We claim that in fact $V_{1}$ is a minimum vertex cover and that $M$ is a maximum matching of $G$. Suppose that $M^{\prime}$ is a maximum matching of $G$ and $\left|M^{\prime}\right|>|M|$. Since $V_{1}$ is a vertex cover, it picks up at least one endpoint from each edge of $M^{\prime}$. Therefore $\left|V_{1}\right|=|M| \geq\left|M^{\prime}\right|$, a contradiction. Therefore $M$ is indeed a maximum matching of $G$ and since any vertex cover of $G$ has size at least $|M|$, it follows that $V_{1}$ is a minimum vertex cover of $G$.

One can test whether the graph is König using the following lemma.

Lemma 3. [12] Given a graph $G$ on $n$ vertices and $m$ edges and a maximum matching of $G$, one can test whether $G$ is König in time $O(n+m)$. If $G$ is indeed König then one can find a minimum vertex cover of $G$ in this time.

Since a maximum matching can be obtained in time $O(m \sqrt{n})$ [34], we have

Lemma 4. Let $G$ be a graph on $n$ vertices and $m$ edges. One can check in time $O(m \sqrt{n})$ whether $G$ is König and, if König, find a bipartition of $V(G)$ into $V_{1} \uplus V_{2}$ with $V_{1}$ a minimum vertex cover of $G$ such that there exists a matching across the cut $\left(V_{1}, V_{2}\right)$ saturating every vertex of $V_{1}$.

\section{The Above Guarantee Vertex Cover Problem}

In this section we show that Above Guarantee Vertex Cover is fixed-parameter tractable and discuss its approximability. This problem plays a central role in this paper and the results established here are used in studying the parameterized complexity and approximability of other König subgraph problems.

Given a graph $G$ it is clear that $\beta(G) \geq \mu(G)$. Recall the definition of Above Guarantee VERTEX COVER: given a graph $G$ and a nonnegative integer parameter $k$ decide whether $\beta(G) \leq$ $\mu(G)+k$. We first show that for the parameterized complexity of the Above GuaranteE VERTEX COVER problem we may, without loss of generality, assume that the input graph has a perfect matching.

Let $G=(V, E)$ be an undirected graph and let $M$ be a maximum matching of $G$. Construct $G^{\prime}=\left(V^{\prime}, E^{\prime}\right)$ as follows. Define

$$
\begin{aligned}
I & =V \backslash V[M] \\
V^{\prime} & =V \cup\left\{u^{\prime}: u \in I\right\} \\
E^{\prime} & =E \cup\left\{\left\{u^{\prime}, v\right\}:\{u, v\} \in E\right\} \cup\left\{\left\{u, u^{\prime}\right\}: u \in I\right\} .
\end{aligned}
$$

Then $M^{\prime}=M \cup\left\{\left\{u, u^{\prime}\right\}: u \in I\right\}$ is a perfect matching for $G^{\prime}$. Note that $\left|V\left(G^{\prime}\right)\right| \leq 2|V(G)|$ and $\left|E\left(G^{\prime}\right)\right| \leq 2|E(G)|+|V(G)|$. 
Lemma 5. Let $G$ be a graph without a perfect matching and let $G^{\prime}$ be the graph obtained by the above construction. Then $G$ has a vertex cover of size $\mu(G)+k$ if and only if $G^{\prime}$ has a vertex cover of size $\mu\left(G^{\prime}\right)+k$.

Proof. Let $M$ denote a maximum matching of $G, I$ denote the set $V(G) \backslash V[M]$ and $I^{\prime}$ denote the new set of vertices that are added in constructing $G^{\prime}$. Clearly, $\mu\left(G^{\prime}\right)=\mu(G)+|I|$.

$(\Rightarrow)$ Let $C$ be a vertex cover of $G$ of size $\mu(G)+k$. Define $C^{\prime}=C \cup I^{\prime}$. It is easy to see that $C^{\prime}$ covers all the edges of $G^{\prime}$. Also, $\left|C^{\prime}\right|=\mu(G)+k+\left|I^{\prime}\right|=\mu\left(G^{\prime}\right)+k$.

$(\Leftarrow)$ Let $C^{\prime}$ be a vertex cover of $G^{\prime}$ of size $\mu\left(G^{\prime}\right)+k$. Define $M^{\prime}$ to be the set of edges of the form $\left\{\left\{u, u^{\prime}\right\}: u \in I\right.$ and $\left.u^{\prime} \in I^{\prime}\right\}$ such that both endpoints are in $C^{\prime}$. One can show that $C=\left(C^{\prime} \cap V[M]\right) \cup\left\{u \in I:\left\{u, u^{\prime}\right\} \in M^{\prime}\right\}$ is a vertex cover of $G$ of size $\mu(G)+k$.

\subsection{Parameterized Complexity}

We show that Above Guarantee Vertex Cover is fixed-parameter tractable by exhibiting a fixed-parameter reduction from Above Guarantee Vertex Cover to a problem know as Min 2-CnF SAT Del [22]. This problem is defined as follows: given a 2-CNF formula and a nonnegative integer $k$, do there exist at most $k$ clauses whose deletion makes the resulting formula satisfiable? This problem is NP-complete and its parameterized complexity was open for quite some time. Recently Razgon and O'Sullivan have shown this problem to be fixed-parameter tractable [29].

Lemma 6. [29] Given a 2-CNF SAT formula $F$ on $n$ variables and $m$ clauses and a nonnegative integer $k$, one can decide whether $F$ has at most $k$ clauses whose deletion makes it satisfiable in time $O\left(15^{k} \cdot k \cdot m^{3}\right)$. That is, the Min 2-CNF SAT DEL problem is fixed-parameter tractable with respect to parameter $k$.

We now describe the reduction from Above Guarantee Vertex Cover to Min 2-Cnf Sat Del (see [4]). Let $G=(V, E)$ be a graph with a perfect matching $P$. For every vertex $u \in V$, define $x_{u}$ to be a Boolean variable. Let $\mathcal{F}(G, P)$ denote the Boolean formula

$$
\mathcal{F}(G, P)=\bigwedge_{(u, v) \in P}\left(\bar{x}_{u} \vee \bar{x}_{v}\right) \bigwedge_{(u, v) \in E}\left(x_{u} \vee x_{v}\right)
$$

Note that $\mathcal{F}(G, P)$ is a formula on $|V|$ variables and at most $2|E|$ clauses.

The proof of the next lemma follows from that of Theorem 5.1 in [4].

Lemma 7. Let $G=(V, E)$ be an n-vertex graph with a perfect matching $P$. Then $G$ has a vertex cover of size at most $n / 2+k$ if and only if there exists an assignment that satisfies all but at most $k$ clauses of $\mathcal{F}(G, P)$.

From the proof of Theorem 5.1 in [4], it also follows that given an assignment that satisfies all but at most $k$ clauses of $\mathcal{F}(G, P)$ one can find (in polynomial time) an assignment that satisfies all but at most $k$ clauses of the form $\left(\bar{x}_{u} \vee \bar{x}_{v}\right)$, where $(u, v) \in P$, that is, clauses that correspond to the perfect matching.

Since Min 2-CnF SAT Del can be solved in time $O\left(15^{k} \cdot k \cdot m^{3}\right)$, where $m$ is the number of clauses in the input formula, we have

Theorem 1. Given a graph $G=(V, E)$ and a nonnegative integer parameter $k$, one can decide whether $\beta(G) \leq \mu(G)+k$ in time $O\left(15^{k} \cdot k \cdot|E|^{3}\right)$. Moreover if $G$ has a vertex cover of size $\mu(G)+k$ then one can find a vertex cover of this size within this time. 
Algo-Above-GuAR-VERTEX-COVER

Input: A graph $G=(V, E)$.

Output: A vertex cover of $G$ of size at most $\mu+O(\log |V| \log \log |V|)(\beta-\mu)$.

1. If $G$ does not have a perfect matching, construct $G^{\prime}$ as in Theorem 5 and set $H \leftarrow G^{\prime}$; else set $H \leftarrow G$.

2. Find a perfect matching $P$ of $H$ and construct $\mathcal{F}(H, P)$. If $G$ did not have a perfect matching then $P$ is the perfect matching obtained from some maximum matching $M$ of $G$ as described in the construction before Theorem 5 .

3. Use the approximation algorithm for Min 2-CNF SAT DeL to obtain an $O(\log n \log \log n)$-approximate solution $\mathcal{S}$ for $\mathcal{F}(H, P)$, where $n=|V(H)|$.

4. Obtain a minimum vertex cover $C$ of the König graph $H \backslash V(\mathcal{S})$, where $V(\mathcal{S})$ is the set of vertices of $H$ corresponding to $\mathcal{S}$, that is, $V(\mathcal{S})$ is the set of the endpoints of those edges in $P$ which correspond to clauses in $\mathcal{S}$.

5. If $H=G$ then return $C \cup V(\mathcal{S})$; else return $(V(\mathcal{S}) \cap V(G)) \cup(V(M) \cap C)$.

Fig. 1. Approximation algorithm for Above Guarantee Vertex Cover.

\subsection{Approximation Algorithm}

The parameterized version of Above Guarantee Vertex Cover asks whether $\tau(G) \leq k$. The optimization version of Above Guarantee Vertex Cover is the problem of finding the minimum value of $\tau(G)$. Therefore an approximation algorithm for ABove GuARAnteE VERTEX COVER approximates the "above-guarantee parameter" rather than the entire vertex cover.

Klein et al. [18] have shown that Min 2-CNF SAT DEL admits a factor- $O(\log n \log \log n)$ approximation algorithm, where $n$ is the number of variables in the 2-SAT formula.

Lemma 8. $[1,18]$ Let $F$ be an instance of Min 2-CNF SAT DEL with $n$ variables. One can in polynomial time obtain a solution that is $O(\log n \log \log n)$ times an optimal solution size. If we are willing to allow randomness, we can obtain a solution that is $O(\sqrt{\log n})$ times an optimal solution size.

We use this algorithm and the reduction from Above Guarantee Vertex Cover to Min 2-CNF SAT Del to obtain an $O(\log n \log \log n)$-approximation algorithm for $\tau(G)$.

An outline of our approximation algorithm is as follows. Given a graph $G=(V, E)$ we first apply the construction described before Theorem 5, if necessary, to obtain a graph $H$ with a perfect matching $P$. Note that $\tau(G)=\tau(H)$. Let $\mathcal{F}(H, P)$ denote the 2-CNF SAT formula obtained from $H$ and $P$ by the construction outlined before Lemma 7 . We noted that given an assignment that satisfies all but at most $k$ clauses of $\mathcal{F}(H, P)$ one can construct an assignment in polynomial time that satisfies all but at most $k$ clauses of the form $\left(\bar{x}_{u} \vee \bar{x}_{v}\right)$, where $(u, v) \in P$. We next use an $O(\log n \log \log n)$ approximation algorithm for Min 2-CNF SAT which "corresponds" to a set $\mathcal{S}$ of edges of the perfect matching $P$. The set $V(\mathcal{S})$, consisting of the endpoints of those edges in $P$ which correspond to clauses in $\mathcal{S}$, represents the vertex cover in excess of the matching size and in the graph $G \backslash V(\mathcal{S})$, the sizes of a minimum vertex cover and maximum matching coincide. That is, $G \backslash V(\mathcal{S})$ is König and therefore by Lemma 4 one can obtain a minimum vertex cover $C$ of this graph in polynomial time. Using $C$ and $\mathcal{S}$, one can reconstruct a vertex cover for $G$ of the appropriate size. The algorithm is presented in Figure 1.

Theorem 2. Let $G$ be a graph on $n$ vertices with a maximum matching of size $\mu$ and a minimum vertex cover of size $\beta$. Then Algo-Above-GuAR-Vertex-Cover finds a vertex cover of $G$ of size $\mu(G)+O(\log n \log \log n)(\beta(G)-\mu(G))$. 
Proof. The proof follows from the fact that the reduction from Above Guarantee Vertex Cover to Min 2-CNF SAT DeL is cost-preserving and that there exists a factor- $O(\log n \log \log n)$ approximation algorithm for the latter.

Thus this algorithm approximates the deficit between the sizes of a minimum vertex cover and a maximum matching. There exists a 2-approximation algorithm for the VERTEX COVER problem which simply includes all vertices of a maximum matching. It is a long standing open problem to devise a polynomial time algorithm which has an approximation factor less than 2 .

Our algorithm is better than any constant factor approximation algorithm for VERTEX Cover whenever

$$
\beta-\mu=o\left(\frac{n}{\log n \log \log n}\right)
$$

and $\mu=\Omega(n)$. To see this, note that a $c$-approximate algorithm, $c>1$, outputs a solution of size $\mu c+(\beta-\mu) c$ whereas our algorithm outputs a solution of size $\mu+O(\alpha(\beta-\mu))$, where $\alpha=\log n \log \log n$. Now if $\beta-\mu=o(n / \alpha)$ and if $\mu=\Omega(n)$, then our algorithm outputs a solution of size $\mu+o(\mu)$, which is better than

$$
\beta c=\mu+\mu(c-1)+(\beta-\mu) c \geq \mu+\Omega(\mu) .
$$

One can obtain a randomized approximation algorithm for $\tau$ using the $O(\sqrt{\log n})$-randomized approximation algorithm for Min 2-CNF SAT DEL, mentioned in Lemma 8, in Step 3 of the algorithm.

\subsection{Hardness of Approximation}

We now show that Above Guarantee Vertex Cover and Min 2-Cnf Sat Del do not admit constant-factor approximation algorithms if the Unique Games Conjecture (UGC) [16] is true. In what follows, we use the abbreviation VC-PM for the VERTEX COVER problem on graphs with a perfect matching.

We make use of the following two results:

Lemma 9. [17] If UGC is true then VERTEX COVER cannot be approximated to within a factor of $2-\epsilon$, for any constant $\epsilon>0$.

Lemma 10. [4,35] If there exists a $(2-\epsilon)$-approximation algorithm for VC-PM then there exists a $(2-\epsilon / 2)$-approximation algorithm for VERTEX COVER.

We can now prove the following.

Theorem 3. Assuming UGC to be true, the Above Guarantee Vertex Cover problem in graphs with a perfect matching cannot be approximated to within a factor of $c$, for any constant $c>1$.

Proof. Suppose that there exists a $c$-approximate algorithm $\mathcal{A}$ for Above Guarantee Vertex Cover on graphs with a perfect matching for some constant $c>1$. By Lemmas 9 and 10, it is sufficient to exhibit a $(2-\epsilon)$-approximate algorithm, for some constant $\epsilon>0$, for VC-PM. This would give us the desired contradiction. We show that $\mathcal{A}$ itself is such an algorithm and obtains a $(2-\epsilon)$-approximate solution with $\epsilon=2 /(c+1)$. 
Let $G=(V, E)$ be a graph on $2 n$ vertices with a perfect matching and minimum vertex cover of size $n+\alpha n$, where $1 / n \leq \alpha \leq 1$. Use algorithm $\mathcal{A}$ on $G$ to obtain a vertex cover of size at most $n+c \alpha n$. The quality of this solution is $(n+c \alpha n) /(n+\alpha n)=(1+c \alpha) /(1+\alpha)$. We distinguish two cases: (1) $\alpha<1 / c$ and (2) $\alpha \geq 1 / c$. We claim that in either case the approximation factor is $2-2 /(c+1)=2 c /(c+1)$. To see this, first consider the case when $\alpha<1 / c$. It is straightforward to show that $(1+c \alpha) /(1+\alpha)<2 c /(c+1)$ if and only if $\alpha<1 / c$. When $\alpha \geq 1 / c$, note that $\mathcal{A}$ can even return the entire vertex set of $G$ as solution. The approximation factor in this case is actually seen to be at most $2 /(1+\alpha)$ which can be easily seen to be at most $2 c /(1+c)$. This completes the proof of the theorem.

Since there is an approximation-preserving reduction (Lemma 7) from Above Guarantee Vertex Cover to Min 2-CNF SAT Del, a constant-factor approximation algorithm for the latter implies the existence of a constant-factor approximation algorithm for the former. Thus we have,

Corollary 1. If UGC is true then MIN 2-CNF SAT DEL does not admit a constant-factor approximation algorithm.

To the best of our knowledge, the only other hardness result known for Min 2-CNF SAT DEL is a 2.88-approximation hardness assuming $\mathrm{P} \neq \mathrm{NP}$ due to Chlebik and Chlebikova [3].

Dinur and Safra [7] have shown that unless $\mathrm{P}=\mathrm{NP}$, VerTex Cover cannot be approximated to within 1.3606 even on graphs with a perfect matching. Using this, we obtain:

Corollary 2. Under the hypothesis $P \neq N P$, ABove GuARAntee Vertex Cover in graphs with a perfect matching cannot be approximated to within 1.7212 .

Proof. Let $\mathcal{A}$ be a $d$-approximation algorithm for computing $\tau(G)$ in graphs with a perfect matching. Let $G$ be an $n$-vertex graph with a perfect matching. Using $\mathcal{A}$, one can obtain a vertex cover of size at most $n / 2+d \tau(G)$. An optimum vertex cover of $G$ has size $n / 2+\tau(G)$. By the NPhardness of approximating VERTEx COVER [7], we must have $(n+2 d \tau(G)) /(n+2 \tau(G)) \geq 1.3606$. Simplifying this yields $n / \tau(G) \leq 2(d-1.3606) / 0.3606$. Note that $n / \tau(G) \geq 2$ and so $d \geq 1.7212$.

\section{The König Vertex Deletion Problem}

Recall that the KÖNIG VERTEX DELETION problem is, given a graph and a nonnegative integer parameter $k$, to decide whether there exist at most $k$ vertices whose deletion makes the resulting graph König. We first investigate the parameterized complexity of this problem and then describe an approximation algorithm for its optimization version.

\subsection{Parameterized Complexity}

We first consider the case when the input graph has a perfect matching.

Graphs with a Perfect Matching. For graphs with a perfect matching it turns out that König Vertex Deletion and Above Guarantee Vertex Cover are fixed-parameter equivalent. 
Lemma 11. Let $G$ be an $n$-vertex graph with a perfect matching. Then $G$ has a vertex cover of size at most $n / 2+k$ if and only if $G$ has a König vertex deletion set of size at most $2 k$.

Proof. $(\Rightarrow)$ Let $P$ be a perfect matching of $G$ and $C$ a vertex cover of $G$ of size at most $n / 2+k$. Consider the subset $M \subseteq P$ of matching edges both of whose endpoints are in $C$. Clearly $V[M]$ is a König vertex deletion set of $G$ of size at most $2 k$.

$(\Leftarrow)$ Conversely let $K$ be a König vertex deletion set of $G$ of size $r \leq 2 k$. Then $G \backslash K$ is a König graph on $n-r$ vertices and hence has a vertex cover $C^{\prime}$ of size at most $(n-r) / 2$. Clearly $C=C^{\prime} \cup K$ is a vertex cover of $G$ of size $\left|C^{\prime}\right|+|K| \leq(n-r) / 2+r=(n+r) / 2 \leq n / 2+k$.

The following corollary follows from Lemmas 5 and 11 and the fact that VERTEx Cover is NP-complete.

Corollary 3. The KÖNIG Vertex Deletion problem is NP-complete.

By Theorem 1, Above Guarantee Vertex Cover is fixed-parameter tractable and therefore we have:

Corollary 4. The KÖNIG VERTEX Deletion problem, parameterized by the solution size, is fixed-parameter tractable on graphs with a perfect matching.

The next result relates the size of a minimum vertex cover with that of a minimum König vertex deletion set for graphs with a perfect matching.

Corollary 5. Let $G$ be an $n$-vertex graph with a perfect matching $P$. Then $\beta(G)=n / 2+k$ if and only if $\kappa(G)=2 k$. Moreover if $\kappa(G)=2 k$, then there exists an edge subset $M \subseteq P$ of size $k$ such that $V[M]$ is a minimum König vertex deletion set of $G$.

If we let $\tau(G)=\beta(G)-\mu(G)$, then the above corollary states: $\kappa(G)=2 \tau(G)$.

Graphs Without a Perfect Matching. For graphs without a perfect matching we do not know of a reduction from König Vertex Deletion to Above Guarantee Vertex Cover and neither does the general case seem reducible to the case where the graph has a perfect matching. However we show that the general problem is fixed-parameter tractable using some new structural results between maximum matchings and vertex covers.

To begin with, we derive a weaker version of Lemma 11 which relates the size of a vertex cover with that of a König vertex deletion set for graphs without a perfect matching.

Lemma 12. Let $G$ be a graph without a perfect matching. If $G$ has a vertex cover of size $\mu(G)+k$ then $G$ has a König vertex deletion set of size at most $2 k$. Moreover, $\tau(G) \leq \kappa(G) \leq 2 \tau(G)$, where $\tau(G)=\beta(G)-\mu(G)$.

Proof. Let $M$ be a maximum matching of $G$ and let $C$ be a vertex cover of $G$ of size $\mu(G)+k$. Define $I=V \backslash V[M], C_{I}=C \cap I$ and $M^{\prime}$ to be the subset of $M$ both of whose endpoints are in $C$. Clearly $V\left[M^{\prime}\right] \cup C_{I}$ is a König vertex deletion set of $G$ of size at most $2 k$. This shows that $\kappa(G) \leq 2 \tau(G)$. To prove that $\tau(G) \leq \kappa(G)$, suppose that there exists $S \subseteq V,|S|<\tau(G)$, such that $G \backslash S$ is König. Then the following easily verifiable inequalities:

$$
\begin{aligned}
& \mu(G \backslash S) \leq \mu(G) \\
& \beta(G \backslash S) \geq \beta(G)-|S|=\mu(G)+\tau(G)-|S|
\end{aligned}
$$

imply that $\beta(G \backslash S)>\mu(G \backslash S)$, a contradiction. 
Suppose $Y$ is a vertex cover in a graph $G=(V, E)$. Consider a maximum matching $M$ between $Y$ and $V \backslash Y$. If $M$ saturates every vertex of $Y$ then the graph is König. If not, then $Y \backslash V(M)$, the set of vertices of $Y$ unsaturated by $M$, is a König vertex deletion set by Lemma 2. What we prove in this section is that if $Y$ is a minimum vertex cover, then $Y \backslash V(M)$ is a minimum König vertex deletion set. Our first observation is that any minimum König vertex deletion set is contained in some minimum vertex cover.

Lemma 13. Let $G$ be a graph with a minimum König vertex deletion set $K$. Let $V(G \backslash K)=V_{1} \uplus$ $V_{2}$ where $V_{2}$ is independent and there is a matching $M$ from $V_{1}$ to $V_{2}$ saturating $V_{1}$. Then $V_{1} \cup K$ is a minimum vertex cover for $G$.

Proof. Suppose $S$ is a vertex cover of $G$ such that $|S|<\left|V_{1}\right|+|K|$. We will show that there exists a König vertex deletion set of size smaller than $|K|$, contradicting our hypothesis. Define $V_{1}^{\prime}=$ $V_{1} \cap S, V_{2}^{\prime}=V_{2} \cap S$ and $K^{\prime}=K \cap S$. Let $A_{1}$ be the vertices of $V_{1}^{\prime}$ whose partner in $M$ is in $V_{2}^{\prime}$ and let $A_{2}$ be the vertices of $V_{1}^{\prime}$ whose partner in $M$ is not in $V_{2}^{\prime}$. See Figure 2. We

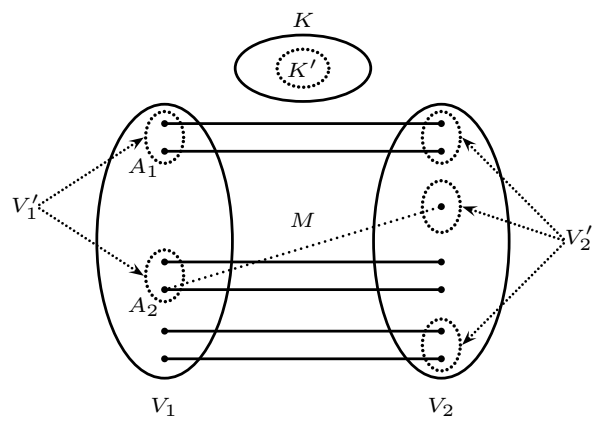

Fig. 2. The sets that appear in the proof of Theorem 13. The matching $M$ consists of the solid edges across $V_{1}$ and $V_{2}$.

claim that $A_{1} \cup K^{\prime}$ is a König vertex deletion set of $G$ and $\left|A_{1} \cup K^{\prime}\right|<|K|$, which will produce the required contradiction and prove the lemma. This claim is proved using the following three claims:

Claim 1. $\left|A_{1} \cup K^{\prime}\right|<|K|$.

Claim 2. $A_{2} \cup V_{2}^{\prime}$ is a vertex cover in $G \backslash\left(A_{1} \cup K^{\prime}\right)$.

Claim 3. There exists a matching between $A_{2} \cup V_{2}^{\prime}$ and $V \backslash\left(V_{1}^{\prime} \cup K^{\prime} \cup V_{2}^{\prime}\right)$ saturating every vertex of $A_{2} \cup V_{2}^{\prime}$.

Proof of Claim 1. Clearly $|S|=\left|V_{1}^{\prime}\right|+\left|V_{2}^{\prime}\right|+\left|K^{\prime}\right|$. Note that $S$ intersects $\left|A_{1}\right|$ of the edges of $M$ in both end points and $|M|-\left|A_{1}\right|$ edges of $M$ in one end point (in either $V_{1}^{\prime}$ or $V_{2}^{\prime}$ ). Furthermore $V_{2}^{\prime}$ has $\left|V_{2}^{\prime} \backslash V(M)\right|$ vertices of $S$ that do not intersect any edge of $M$. Hence $|M|+\left|A_{1}\right|+\left|V_{2}^{\prime} \backslash V(M)\right|=$ $\left|V_{1}^{\prime}\right|+\left|V_{2}^{\prime}\right|$. That is, $\left|V_{1}^{\prime}\right|+\left|V_{2}^{\prime}\right|=\left|V_{1}\right|+\left|A_{1}\right|+\left|V_{2}^{\prime} \backslash V(M)\right|$ (as $|M|=\left|V_{1}\right|$ ). Hence $|S|<\left|V_{1}\right|+|K|$ implies that $\left|A_{1}\right|+\left|V_{2}^{\prime} \backslash V(M)\right|+\left|K^{\prime}\right|<|K|$ which implies that $\left|A_{1}\right|+\left|K^{\prime}\right|<|K|$ proving the claim.

Proof of Claim 2. Since $S=A_{1} \cup A_{2} \cup V_{2}^{\prime} \cup K^{\prime}$ is a vertex cover of $G$, clearly $A_{2} \cup V_{2}^{\prime}$ covers all edges in $G \backslash\left(A_{1} \cup K^{\prime}\right)$. 
Proof of Claim 3. Since the partner of a vertex in $A_{2}$ in $M$ is in $V \backslash\left(V_{1}^{\prime} \cup K^{\prime} \cup V_{2}^{\prime}\right)$, we can use the edges of $M$ to saturate vertices in $A_{2}$. To complete the proof, we show that in the bipartite graph $\left(V_{2}^{\prime},\left(V_{1} \backslash V_{1}^{\prime}\right) \cup\left(K \backslash K^{\prime}\right)\right)$ there is a matching saturating $V_{2}^{\prime}$. To see this, note that any subset $D \subseteq V_{2}^{\prime}$ has at least $|D|$ neighbors in $\left(V_{1} \backslash V_{1}^{\prime}\right) \cup\left(K \backslash K^{\prime}\right)$. For otherwise, let $D^{\prime}$ be the set of neighbors of $D$ in $\left(V_{1} \backslash V_{1}^{\prime}\right) \cup\left(K \backslash K^{\prime}\right)$ where we assume $|D|>\left|D^{\prime}\right|$. Then $(S \backslash D) \cup D^{\prime}$ is a vertex cover of $G$ of size strictly less than $|S|$, contradicting the fact that $S$ is a minimum vertex cover. To see that $(S \backslash D) \cup D^{\prime}$ is indeed a vertex cover of $G$, note that $S \backslash V_{2}^{\prime}$ covers all edges of $G$ except those in the graph $\left(V_{2}^{\prime},\left(V_{1} \backslash V_{1}^{\prime}\right) \cup\left(K \backslash K^{\prime}\right)\right)$ and all these edges are covered by $\left(V_{2}^{\prime} \backslash D\right) \cup D^{\prime}$. Hence by Hall's theorem, there exists a matching saturating all vertices of $V_{2}^{\prime}$ in the bipartite graph $\left(V_{2}^{\prime},\left(V_{1} \backslash V_{1}^{\prime}\right) \cup\left(K \backslash K^{\prime}\right)\right)$, proving the claim.

This completes the proof of the lemma.

Lemma 13 has interesting consequences.

Corollary 6. For any two minimum König vertex deletion sets $K_{1}$ and $K_{2}, \mu\left(G \backslash K_{1}\right)=\mu(G \backslash$ $\left.K_{2}\right)$.

Proof. Since $K_{1}$ and $K_{2}$ are minimum König vertex deletion sets of $G, \beta\left(G \backslash K_{1}\right)=\mu\left(G \backslash K_{1}\right)$ and $\beta\left(G \backslash K_{2}\right)=\mu\left(G \backslash K_{2}\right)$. By Theorem 13, $\beta\left(G \backslash K_{1}\right)+\left|K_{1}\right|=\beta(G)$ and $\beta\left(G \backslash K_{2}\right)+\left|K_{2}\right|=\beta(G)$. Since $\left|K_{1}\right|=\left|K_{2}\right|$, it follows that $\beta\left(G \backslash K_{1}\right)=\beta\left(G \backslash K_{2}\right)$ and hence $\mu\left(G \backslash K_{1}\right)=\mu\left(G \backslash K_{2}\right)$.

From Lemma 4 and 13, we get

Corollary 7. Given a graph $G=(V, E)$ and a minimum König vertex deletion set for $G$, one can construct a minimum vertex cover for $G$ in polynomial time.

Our goal now is to prove the "converse" of Corollary 7. In particular, we would like to construct a minimum König vertex deletion set from a minimum vertex cover. Our first step is to show that if we know that a given minimum vertex cover contains a minimum König vertex deletion set then we can find the König vertex deletion set in polynomial time. Recall that given a graph $G=(V, E)$ and $A, B \subseteq V$ such that $A \cap B=\emptyset$, we use $\mu(A, B)$ to denote a maximum matching in the bipartite graph comprising of the vertices in $A \cup B$ and the edges in $\{\{u, v\} \in E: u \in A, v \in B\}$. We denote this graph by $(A, B)$.

Lemma 14. Let $K$ be a minimum König vertex deletion set and $Y$ a minimum vertex cover of a graph $G=(V, E)$ such that $K \subseteq Y$. Then $\mu(G \backslash K)=\mu(Y, V \backslash Y)$ and $|K|=|Y|-\mu(Y, V \backslash Y)$.

Proof. If $G$ is König then the theorem clearly holds. Therefore assume that $K \neq \emptyset$. Note that $Y \backslash$ $K$ is a minimum vertex cover of the König graph $G \backslash K$. Thus $\mu(G \backslash K)=\mu(Y \backslash K, V \backslash Y)$. We claim that $\mu(Y \backslash K, V \backslash Y)=\mu(Y, V \backslash Y)$. For if not, we must have $\mu(Y \backslash K, V \backslash Y)<\mu(Y, V \backslash Y)$. Then let $M$ be a maximum matching in the bipartite graph $(Y, V \backslash Y)$ and $K^{\prime} \subseteq Y$ be the set of vertices unsaturated by $M$. Note that $K^{\prime} \neq \emptyset$ is a König vertex deletion set for $G$. Since $\mu(Y, V \backslash Y)=|Y|-\left|K^{\prime}\right|$ and $\mu(Y \backslash K, V \backslash Y)=|Y|-|K|$ we have $\left|K^{\prime}\right|<|K|$, a contradiction, since by hypothesis $K$ is a smallest König vertex deletion set for $G$. Therefore we must have $\mu(G \backslash K)=\mu(Y, V \backslash Y)$ and $|K|=|Y|-\mu(Y, V \backslash Y)$.

The next lemma says that $\mu(Y, V \backslash Y)$ is the same for all minimum vertex covers $Y$ of a graph $G$. Together with Lemma 14, this implies that if $K$ is a minimum König vertex deletion set and $Y$ is a minimum vertex cover of a graph $G=(V, E)$, then $\mu(G \backslash K)=\mu(Y, V \backslash Y)$. 
Lemma 15. For any two minimum vertex covers $Y_{1}$ and $Y_{2}$ of $G, \mu\left(Y_{1}, V \backslash Y_{1}\right)=\mu\left(Y_{2}, V \backslash Y_{2}\right)$.

Proof. Suppose without loss of generality that $\mu\left(Y_{1}, V \backslash Y_{1}\right)>\mu\left(Y_{2}, V \backslash Y_{2}\right)$. Let $M_{1}$ be a maximum matching in the bipartite graph $\left(Y_{1}, V \backslash Y_{1}\right)$. To arrive at a contradiction, we study how $Y_{2}$ intersects the sets $Y_{1}$ and $V \backslash Y_{1}$ with respect to the matching $M_{1}$. To this end, we define the following sets (see Figure 3):

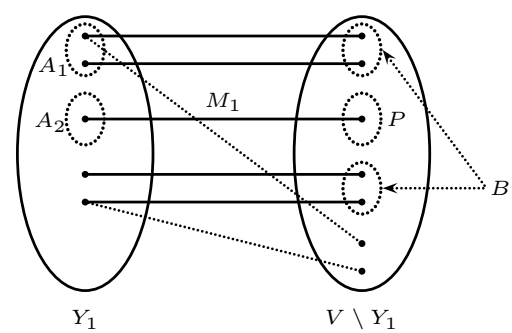

Fig. 3. The sets that appear in the proof of Lemma 15. The solid edges across $Y_{1}$ and $V \backslash Y_{1}$ constitute the matching $M_{1}$.

$-A=Y_{2} \cap Y_{1} \cap V\left(M_{1}\right)$.

$-B=Y_{2} \cap\left(V \backslash Y_{1}\right) \cap V\left(M_{1}\right)$.

- $A_{1}$ is the set of vertices in $A$ whose partners in $M_{1}$ are also in $Y_{2}$.

- $A_{2}$ is the set of vertices in $A$ whose partners in $M_{1}$ are not in $Y_{2}$.

We first show that

Claim. In the bipartite graph $\left(Y_{2}, V \backslash Y_{2}\right)$ there is a matching saturating each vertex in $A_{2} \cup B$.

It will follow from the claim that $\mu\left(Y_{2}, V \backslash Y_{2}\right) \geq\left|A_{2}\right|+|B|$. However, note that $Y_{2}$ intersects every edge of $M_{1}$ at least once (as $Y_{2}$ is a vertex cover). More specifically, $Y_{2}$ intersects $\left|A_{1}\right|$ edges of $M_{1}$ twice and $\left|M_{1}\right|-\left|A_{1}\right|$ edges once (either in $Y_{1}$ or in $V \backslash Y_{1}$ ). Hence, $|A|+|B|=\left|M_{1}\right|+\left|A_{1}\right|$ and so $\left|A_{2}\right|+|B|=\left|M_{1}\right|$ and so $\mu\left(Y_{2}, V \backslash Y_{2}\right) \geq\left|A_{2}\right|+|B|=\left|M_{1}\right|$ a contradiction to our assumption at the beginning of the proof. Thus it suffices to prove the claim.

Proof of Claim. Let $P$ denote the partners of the vertices of $A_{2}$ in $M_{1}$. Since $P \subseteq V \backslash Y_{2}$, we use the edges of $M_{1}$ to saturate vertices of $A_{2}$. Hence it is enough to show that the bipartite graph $\mathcal{B}=\left(B,\left(V \backslash Y_{2}\right) \backslash P\right)$ contains a matching saturating the vertices in $B$. Suppose not. By Hall's Theorem there exists a set $D \subseteq B$ such that $\left|N_{\mathcal{B}}(D)\right|<|D|$. We claim that the set $Y_{2}^{\prime}:=Y_{2} \backslash D+N_{\mathcal{B}}(D)$ is a vertex cover of $G$. To see this, note that the vertices in $Y_{2} \backslash D$ cover all the edges of $G$ except those in the bipartite graph $\left(D, Y_{1} \cap\left(V \backslash Y_{2}\right)\right)$ and these are covered by $N_{\mathcal{B}}(D)$. Therefore $Y_{2}^{\prime}$ is a vertex cover of size strictly smaller than $Y_{2}$, a contradiction. This proves that there exists a matching in $\left(Y_{2}, V \backslash Y_{2}\right)$ saturating each vertex in $A_{2} \cup B$.

This completes the proof of the lemma.

The next two lemmas show how we can obtain a minimum König vertex deletion set from a minimum vertex cover in polynomial time. 
Lemma 16. Given a graph $G=(V, E)$, let $Y$ be any minimum vertex cover of $G$ and $M$ a maximum matching in the bipartite graph $(Y, V \backslash Y)$. Then $K:=Y \backslash V(M)$ is a minimum König vertex deletion set of $G$.

Proof. Clearly $K$ is a König vertex deletion set. Let $K_{1}$ be a minimum König vertex deletion set of $G$. By Lemma 13, there exists a minimum vertex cover $Y_{1}$ such that $K_{1} \subseteq Y_{1}$ and

$$
\begin{aligned}
\left|K_{1}\right| & =\left|Y_{1}\right|-\mu\left(Y_{1}, V \backslash Y_{1}\right)(\text { By Lemma } 14 .) \\
& =|Y|-\mu\left(Y_{1}, V \backslash Y_{1}\right) \quad\left(\text { Since } Y_{1} \text { and } Y\right. \text { are minimum vertex covers.) } \\
& =|Y|-\mu(Y, V \backslash Y) \quad(\text { By Lemma } 15 .) \\
& =|K|
\end{aligned}
$$

This proves that $K$ is a minimum König vertex deletion set.

Lemma 17. Given a graph $G=(V, E)$ and a minimum vertex cover for $G$, one can construct a minimum König vertex deletion set for $G$ in polynomial time.

Note that although both these problems-Vertex Cover and König Vertex Deletionare NP-complete, we know of very few pairs of such parameters where we can obtain one from the other in polynomial time on the same graph (e.g. edge dominating set and minimum maximal matching, see [13]). In fact, there are parameter pairs such as dominating set and vertex cover where such a polynomial-time transformation is not possible unless $\mathrm{P}=\mathrm{NP}$. This follows since in bipartite graphs, for instance, a minimum vertex cover is computable in polynomial time whereas computing a minimum dominating set is NP-complete.

We are now ready to prove that the KÖNIG VERTEX DELETION problem is fixed-parameter tractable in general graphs.

Theorem 4. Given a graph $G=(V, E)$ and an integer parameter $k$, the problem of whether $G$ has a subset of at most $k$ vertices whose deletion makes the resulting graph König can be decided in time $O\left(15^{k} \cdot k^{2} \cdot|E|^{3}\right)$. Hence the KÖNIG VERTEX DeLeTION problem is fixed-parameter tractable when parameterized by the solution size.

Proof. Use the FPT algorithm from Theorem 1 to test whether $G$ has a vertex cover of size at most $\mu(G)+k$. If not, by Lemma 12 , we know that the size of a minimum König vertex deletion set is strictly more than $k$. Therefore return NO. If yes, then find the size of a minimum vertex cover by applying Theorem 1 with every integer value between 0 and $k$ for the excess above $\mu(G)$. Note that for yes-instances of the Above Guarantee Vertex Cover problem, the FPT algorithm actually outputs a vertex cover of size $\mu(G)+k$. We therefore obtain a minimum vertex cover of $G$. Use Lemma 16 to get a minimum König vertex deletion set in polynomial time and depending on its size answer the question. It is easy to see that all this can be done in time $O\left(15^{k} \cdot k^{2} \cdot|E|^{3}\right)$.

Note that computing a maximum independent set (or equivalently a minimum vertex cover) in an $n$-vertex graph can be done in time $O^{*}\left(2^{0.288 n}\right)$ [10]. By Lemma 17 , one can compute a minimum König vertex deletion set in the same exponential time.

Corollary 8. Given a graph $G=(V, E)$ on $n$ vertices one can find a minimum König vertex deletion set in time $O^{*}\left(2^{0.288 n}\right)=O^{*}\left(1.221^{n}\right) .^{5}$

\footnotetext{
${ }^{5}$ The $O^{*}$ notation suppresses polynomial terms.
} 
Suppose we wanted to compute a minimum König vertex deletion set on graphs of treewidth at most $w$. A dynamic programming approach as for Dominating Set or Independent Set is not obvious. However since one can obtain a minimum vertex cover on graphs with treewidth at most $w$ in time $O^{*}\left(2^{w}\right)$ [27], by Lemma 17, one can obtain a minimum König deletion set within this time.

Corollary 9. If a tree-decomposition for $G$ of width $w$ is given, one can find a minimum König vertex deletion set in time $O^{*}\left(2^{w}\right)$.

\subsection{Approximability}

In Lemma 12 we established that for any graph $G$ (whether it has a perfect matching or not), $\tau(G) \leq \kappa(G) \leq 2 \tau(G)$, where $\tau(G)=\beta(G)-\mu(G)$ is the excess vertex cover beyond the size of a maximum matching. Therefore a good approximation of the above guarantee parameter $\tau$ yields a good approximation for $\kappa$ and vice versa.

In the algorithm outlined in Figure 1, note that $V(\mathcal{S}) \cap V(G)$ is actually a König vertex deletion set of $G$. Since $|V(\mathcal{S})| \leq O(\log n \log \log n)(\beta-\mu)$, we have, by Theorem 2

Theorem 5. Given a graph $G$ on $n$ vertices, there exists an algorithm that approximates the König vertex deletion set of $G$ to within a factor of $O(\log n \log \log n)$.

On graphs with a perfect matching, the Above Guarantee Vertex Cover and König Vertex Deletion problems are equivalent and hence Theorem 3 and Lemma 11 imply

Corollary 10. If UGC is true then König VERTEX Deletion does not admit a constantfactor approximation algorithm.

Since König Vertex Deletion and Above Guarantee Vertex Cover are equivalent in terms of approximability in graphs with a perfect matching (Lemma 11), Corollary 2 implies

Corollary 11. Under the hypothesis $P \neq N P$, KÖNIG VeRTEx Deletion in graphs with a perfect matching cannot be approximated to within 1.7212 .

\section{The Induced König Subgraph Problem}

In this section we deal with the parameterized complexity and approximability of the vertex and edge versions of the INDUCED KÖNIG SUBGRAPH problem.

\section{$5.1 \quad$ Vertex Induced König Subgraph}

The NP-completeness of this problem follows from that of KÖNIG VERTEX DELETION but it has a different parameterized complexity. We show that VERTEX INDUCED KÖNIG SubGRAPH is $W[1]$-hard and is as hard to approximate as the InDEPENDENT SET problem.

Theorem 6. Vertex Induced KÖNIG Subgraph is $W[1]$-hard with respect to the number of vertices in the induced subgraph as parameter. 
Proof. We give a parameter-preserving reduction from IndePendent Set to VerTex Induced König Subgraph. Given an instance $(G, k)$ of Independent Set, construct a graph $H$ as follows. The vertex set of $H$ consists of two copies of $V(G)$ namely, $V_{1}=\left\{u_{1}: u \in V(G)\right\}$ and $V_{2}=\left\{u_{2}: u \in V(G)\right\}$. For all $u \in V(G),\left(u_{1}, u_{2}\right) \in E(H)$. If $(u, v) \in E(G)$, add the edges $\left(u_{1}, v_{1}\right),\left(u_{2}, v_{2}\right),\left(u_{1}, v_{2}\right)$ and $\left(v_{1}, u_{2}\right)$ in $E(H)$. $H$ has no more edges.

We claim that $G$ has an independent set of size $k$ if and only if $H$ has a König subgraph of size $2 k$. Let $I$ be an independent set of size $k$ in $G$. Let $K=\left\{u_{1}, u_{2} \in V(H): u \in I\right\}$. Clearly $H[K]$ is an induced matching on $2 k$ vertices and is bipartite and hence König. Conversely, let $K$ be a König subgraph of $H$ on $2 k$ vertices. By Lemma 2, every König graph on $n$ vertices has an independent set of size at least $n / 2$. Therefore let $I^{\prime}$ be an independent set of $K$ of size at least $k$. Define $I=\left\{u \in V(G)\right.$ : either $u_{1}$ or $\left.u_{2} \in I^{\prime}\right\}$. It is clear that the vertices of $I^{\prime}$ correspond to distinct vertices of $G$ and hence $|I| \geq k$. It is also easy to see that the vertices in $I$ actually form an independent set in $G$.

Since the IndEPENDENT SET problem can have no approximation algorithms with factor $O\left(n^{1-\epsilon}\right)$, for any $\epsilon>0$, unless $\mathrm{P}=\mathrm{NP}[15,36]$, we have:

Corollary 12. There is no approximation algorithm for VERTEX INDUCED KÖNIG SUBGRAPH with factor $O\left(n^{1-\epsilon}\right)$, for any $\epsilon>0$, unless $P=N P$.

In the reduction above, $|V(H)|=2|V(G)|$ and $(G, k)$ is a yes-instance of INDEPENDENT Set if and only if $(H, 2 k)$ is a yes-instance of Vertex Induced König Subgraph. Thus this reduction can also be viewed as a reduction from VerTex Cover to KöNIG VerTex DeLETION giving yet another proof of Corollary 3 .

\subsection{Edge Induced König Subgraph}

We now show that EdGe Induced König Subgraph is NP-complete and study its approximability and parameterized complexity. We will see that, unlike the other König subgraph problems, EdGe IndUCED KÖNIG SUBGRAPH admits a good (constant-factor) approximation algorithm.

NP-Completeness. Since both König Edge Deletion and Edge Induced König SubGRAPH have the same complexity from the classical point of view, it is sufficient to prove that one of them is NP-complete. We actually show that:

Theorem 7. KÖNIG Edge Deletion is NP-complete.

Proof. We give a reduction from Min 2-CnF SAT Del. Let $\Phi$ be a 2-CNF SAT formula with $m$ clauses composed of the literals $\left\{x_{1}, \bar{x}_{1}, \ldots, x_{n}, \bar{x}_{n}\right\}$. Construct a graph $G_{\Phi}=(V, E)$ as follows. Let

$$
V:=\left\{x_{1}, \bar{x}_{1}, x_{11}, \bar{x}_{11}, \ldots, x_{1, m+2}, \bar{x}_{1, m+2}, \ldots, x_{n}, \bar{x}_{n}, x_{n, 1}, \bar{x}_{n, 1}, \ldots, x_{n, m+2}, \bar{x}_{n, m+2}\right\}
$$

that is, $V$ consists of $m+3$ copies of $x_{i}, \bar{x}_{i}$. Add exactly those edges so that for each $1 \leq i \leq n$, the vertex sets $L_{i}=\left\{x_{i}, x_{i, 1}, \ldots, x_{i, m+2}\right\}$ and $R_{i}=\left\{\bar{x}_{i}, \bar{x}_{i, 1}, \ldots, \bar{x}_{i, m+2}\right\}$ form a complete bipartite graph with $L_{i}$ and $R_{i}$ as the left and right partite sets, respectively. Finally for each clause $\left(y_{i} \vee y_{j}\right)$ of $\Phi$ add an edge $\left(y_{i}, y_{j}\right)$ (among the vertices $\left\{x_{1}, \bar{x}_{1}, \ldots, x_{n}, \bar{x}_{n}\right\}$ ). Note that $G_{\Phi}$ has a perfect matching and that each clause of $\Phi$ corresponds to an edge of $G_{\Phi}$. 
Claim. There exists an assignment satisfying all but $k$ clauses of $\Phi$ if and only if there exist at most $k$ edges whose deletion makes $G_{\Phi}$ König.

$(\Rightarrow)$ Let $\alpha$ be an assignment to the variables of $\Phi$ that satisfies all but $k$ clauses. Each of these $k$ clauses corresponds to a distinct edge in $G_{\Phi}$. Delete these edges from $G_{\Phi}$. Then for each edge in the remaining graph, at least one endpoint of the edge is assigned 1 by the assignment $\alpha$. To prove that the remaining graph is König, by Lemma 2, we must demonstrate a bipartition of the vertex set into $V_{1} \uplus V_{2}$ (say) such that $V_{2}$ is independent and there exists a matching across the cut $\left(V_{1}, V_{2}\right)$ saturating $V_{1}$. If $\alpha\left(x_{i}\right)=1$ then place the vertices $x_{i}, x_{i, 1}, \ldots x_{i, m+2}$ in $V_{1}$; else place $\bar{x}_{i}, \bar{x}_{i, 1}, \ldots \bar{x}_{i, m+2}$ in $V_{1}$. The remaining vertices are placed in $V_{2}$. As $\Phi$ satisfies all remaining clauses, $V_{2}$ is independent. Note that if $x_{i} \in V_{1}$ then $\bar{x}_{i} \in V_{2}$ and vice versa. Also if $x_{i, j} \in V_{1}$ then $\bar{x}_{i, j} \in V_{2}$ and vice versa. Hence there exists a matching across the cut $\left(V_{1}, V_{2}\right)$ that saturates $V_{1}$.

$(\Leftarrow)$ Conversely suppose that deleting a set $S$ of $k$ edges makes $G_{\Phi}$ König. We will assume that $S$ is a minimal edge deletion set. Any minimal König edge deletion set has size at most $m$, since deleting all the $m$ "clause edges" from $G_{\Phi}$ results in a König graph. Therefore we may assume that $k \leq m$. Call the resulting graph $G_{\Phi}^{\prime}$. Then the vertex set of $G_{\Phi}^{\prime}$ can be partitioned into $V_{1}$ and $V_{2}$ such that $V_{2}$ is independent and there exists a matching across the cut $\left(V_{1}, V_{2}\right)$ that saturates $V_{1}$.

Claim 1. For each $1 \leq i \leq n$, it is not the case that $x_{i}, \bar{x}_{i} \in V_{2}$.

Suppose that for some $1 \leq i \leq n, x_{i}, \bar{x}_{i} \in V_{2}$. Then it must be that $\bar{C}_{i}=\left\{\bar{x}_{i, 1}, \ldots, \bar{x}_{i, m+2}\right\} \nsubseteq$ $V_{2}$ since otherwise $m+2$ edges between $x_{i}$ and the vertices of $\bar{C}_{i}$ must have been deleted from $G_{\Phi}$ to obtain $G_{\Phi}^{\prime}$, a contradiction. If $\bar{C}_{i} \subseteq V_{1}$ then $C_{i}=\left\{x_{i, 1}, \ldots, x_{i, m+2}\right\} \subseteq V_{2}$ for there to be a matching across the cut $\left(V_{1}, V_{2}\right)$ saturating all of $\bar{C}_{i}$. But then $m+2$ edges between $\bar{x}_{i}$ and $C_{i}$ must have been deleted from $G_{\Phi}$ to obtain $G_{\Phi}^{\prime}$, again a contradiction. This argument shows that that there exist integers $p, q \geq 1$ with $p+q=m+2$, such that $V_{1}$ contains $p$ vertices of $\bar{C}_{i}$ and $V_{2}$ contains the remaining $q$ vertices of $\bar{C}_{i}$. In order for there to be a matching across $\left(V_{1}, V_{2}\right)$ saturating all $p$ vertices of $\bar{C}_{i}$ in $V_{1}$ there must be at least $p$ vertices of $C_{i}$ in $V_{2}$. Since the vertices of $C_{i}$ and $\bar{C}_{i}$ form a complete bipartite graph we end up deleting at least $p q+1 \geq m+2$ edges of $G_{\Phi}$, a contradiction yet again. This proves Claim 1 .

Claim 2. For each $1 \leq i \leq n$, it is not the case that $x_{i}, \bar{x}_{i} \in V_{1}$.

Suppose that there exists $i, 1 \leq i \leq n$, such that $x_{i}, \bar{x}_{i} \in V_{1}$. Let $M$ be a matching across $\left(V_{1}, V_{2}\right)$ that saturates the vertices of $V_{1}$. We distinguish three cases.

Case 1. $\bar{C}_{i}=\left\{\bar{x}_{i, 1}, \ldots, \bar{x}_{i, m+2}\right\} \subseteq V_{1}$.

This implies that $C_{i}=\left\{x_{i, 1}, \ldots, x_{i, m+2}\right\} \subseteq V_{2}$ as otherwise no matching across $\left(V_{1}, V_{2}\right)$ would saturate all of $\bar{C}_{i}$. Let $x_{a_{1}}$ and $x_{b_{1}}$ be the partners of $x_{i}$ and $\bar{x}_{i}$, respectively, relative to the matching $M$. By Claim $1, x_{a_{1}}$ and $x_{b_{1}}$ represent different variables, that is, they are not the negations of one another. This implies that $\bar{x}_{a_{1}}$ and $\bar{x}_{b_{1}}$ are in $V_{1}$. Consider the pair $x_{a_{1}}, \bar{x}_{a_{1}}$. We will show that $C_{a_{1}}=\left\{x_{a_{1}, 1}, \ldots, x_{a_{1}, m+2}\right\} \subseteq V_{2}$ and $\bar{C}_{a_{1}}=\left\{\bar{x}_{a_{1}, 1}, \ldots, \bar{x}_{a_{1}, m+2}\right\} \subseteq V_{1}$. For if not, suppose that $1 \leq q \leq m+1$ vertices of $\bar{C}_{a_{1}}$ are in $V_{2}$ while the remaining $p \geq 1$ vertices of $\bar{C}_{a_{1}}$ are in $V_{1}$. In order for the vertices of $\bar{C}_{a_{1}}$ to have partners with respect to $M$ at least $p$ vertices of $C_{a_{1}}$ must be in $V_{2}$. This implies that at least $p q$ edges have been deleted from $G_{\Phi}$ 
to obtain $G_{\Phi}^{\prime}$. Since $p+q=m+2$, we have $p q \geq m+1$, a contradiction. The upshot is that the partners of $\bar{x}_{a_{1}}$ and $\bar{x}_{b_{1}}$ relative to $M$ are vertices from the set $\left\{x_{1}, \bar{x}_{1}, \ldots, x_{n}, \bar{x}_{n}\right\}$. Let the partners of $\bar{x}_{a_{1}}$ and $\bar{x}_{b_{1}}$ relative to $M$ be $x_{a_{2}}$ and $x_{b_{2}}$ respectively. Again by Claim $1, x_{a_{2}}$ and $x_{b_{2}}$ represent distinct variables and hence $\bar{x}_{a_{2}}$ and $\bar{x}_{b_{2}}$ are in $V_{1}$. Repeating this argument we obtain a sequence of vertices of the form:

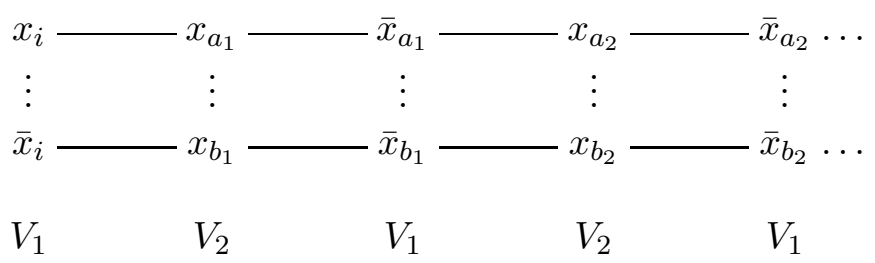

Since there are only $2 n$ vertices such a chain must end at $V_{2}$ with both endpoints being the negation of one another. This contradicts Claim 1 and shows that this situation does not arise.

Case 2. $\bar{C}_{i}=\left\{\bar{x}_{i, 1}, \ldots, \bar{x}_{i, m+2}\right\} \subseteq V_{2}$ and $C_{i}=\left\{x_{i, 1}, \ldots, x_{i, m+2}\right\} \subseteq V_{1}$.

This is the symmetric version of Case 1 and can be handled similarly.

Case 3. For some integers $p, q \geq 1$ and $p+q=m+2, p$ vertices of $\bar{C}_{i}$ lie in $V_{1}$ and the remaining $q$ vertices lie in $V_{2}$.

By symmetry, $p$ vertices of $C_{i}$ must lie in $V_{2}$. This implies that at least $p q \geq m+1$ edges have been deleted from $G_{\Phi}$ to obtain $G_{\Phi}^{\prime}$, a contradiction. This proves Claim 2 .

Since $S$ was assumed to be a minimal König edge deletion set, for each vertex $y_{i}$, all copies $y_{i, 1}, \ldots, y_{i, m+2}$ of it must be placed in the same partition as $y_{i}$ itself and hence all edges of $G_{\Phi}$ that are part of the $n$ copies of $K_{m+3, m+3}$ lie across the cut $\left(V_{1}, V_{2}\right)$. It is easy to see that any other partitioning of the copies would result in more edges being deleted unnecessarily. Therefore the edges that were deleted from $G_{\Phi}$ were those that corresponded to the clauses of $\Phi$. If a vertex $y_{i}$ is in $V_{1}$ assign the corresponding literal the value 1 ; else assign the literal the value 0 . Note that this assignment is consistent as all copies of a vertex are in the same partition as the vertex itself and for no vertex do we have that $x_{i}, \bar{x}_{i} \in V_{1}$ or $x_{i}, \bar{x}_{i} \in V_{2}$. This assignment satisfies all but the $k$ clauses that correspond to the edges that were deleted.

Since the above reduction is cost-preserving, approximation lower-bounds for Min 2-CNF Sat Del carry over to König Edge Deletion. Therefore by Corollary 1, we obtain

Corollary 13. If UGC is true then KÖNIG EDGE DELETION does not have a c-approximation algorithm, for any constant $c>1$.

Chlebik and Chlebikova [3] have shown that it is NP-hard to approximate Min 2-CNF SAT DeL to within $8 \sqrt{5}-15 \approx 2.88$. This gives us

Corollary 14. It is NP-hard to approximate König Edge Deletion to within 2.88.

Approximation Results. For Edge Induced KÖNIG Subgraph, it is easy to obtain a 2approximation algorithm by simply finding a cut of size $m / 2$ and then deleting all the other edges. As the resulting graph will be bipartite it will be König. In this subsection, we give a 4/3approximation algorithm for graphs with a perfect matching and a 5/3-approximation algorithm for general graphs based on the following combinatorial results. 
Lemma 18. Let $G=(V, E)$ be a graph with a maximum matching $M$ and let $G_{M}=\left(V_{M}, E_{M}\right)$ be the graph induced on the vertices $V(M)$ of $M$. Then $G$ has an edge-induced König subgraph of size at least $\frac{3\left|E_{M}\right|}{4}+\frac{\left|E-E_{M}\right|}{2}+\frac{|M|}{4}$.

Proof. Randomly partition the vertex set of $G$ into $V_{1} \uplus V_{2}$ as follows. For each edge $e_{i} \in M$, select an endpoint of $e_{i}$ with probability $1 / 2$ and place it in $V_{1}$. Define $V_{2}=V-V_{1}$. Note that the edges in $M$ always lie across the cut $\left(V_{1}, V_{2}\right)$. An edge of $E_{M}-M$ is in $G\left[V_{2}\right]$ with probability $1 / 4$; an edge in $E-E_{M}$ lies in $G\left[V_{2}\right]$ with probability $1 / 2$. For each edge $e \in E$, define $X_{e}$ to be the indicator random variable that takes the value 1 if $e \in G\left[V_{2}\right]$ and 0 otherwise. Also define $X=\sum_{e \in E} X_{e}$. Then

$$
E[X]=\sum_{e \in E} E\left[X_{e}\right]=\frac{\left|E_{M}-M\right|}{4}+\frac{\left|E-E_{M}\right|}{2} .
$$

Deleting the edges in $G\left[V_{2}\right]$ results in a König graph with

$$
\frac{3\left|E_{M}\right|}{4}+\frac{\left|E-E_{M}\right|}{2}+\frac{|M|}{4}
$$

edges in expectation. This algorithm can be easily derandomized by the method of conditional probabilities (see, for instance [26]). This completes the proof.

If $G=(V, E)$ has a perfect matching $M$ then $E_{M}=E$ and $|M|=|V| / 2$ and we have

Corollary 15. Let $G=(V, E)$ be a graph on $n$ vertices and $m$ edges with a perfect matching. Then $G$ has a subgraph with at least $3 \mathrm{~m} / 4+n / 8$ edges that is König. This subgraph can be found in time $O(m n)$.

Lemma 19. Let $G=(V, E)$ be an undirected graph on $n$ vertices and $m$ edges. Then $G$ has an edge-induced König subgraph of size at least $3 \mathrm{~m} / 5$.

Proof. Let $M$ be a maximum matching of $G$ and let $G\left[V_{M}\right]=\left(V_{M}, E_{M}\right)$ be the subgraph induced by the vertices $V(M)$ of $M$. Let $\eta(G)$ denote the size of the maximum edge induced König subgraph of $G$. By Lemma 18,

$$
\eta(G) \geq \frac{\left|E_{M}\right|+|M|}{4}+\frac{|E|}{2} .
$$

Observe that by deleting all the edges in $G\left[V_{M}\right]$ we obtain a König subgraph of $G$. In fact, this is a bipartite graph with bipartition $V_{M}$ and $V-V_{M}$. Therefore if $\left|E-E_{M}\right| \geq 3 m / 5$, the statement of the lemma clearly holds. Otherwise, $\left|E_{M}\right| \geq 2 m / 5$ and by Lemma 18 , we obtain $\eta(G) \geq|M| / 4+3 m / 5$. This completes the proof.

The following theorem follows from Corollary 15 and Lemma 19 and the fact that the optimum König subgraph has at most $m$ edges.

Theorem 8. The optimization version of EdGE Induced KönIG SUbGRAPH is approximable to within a factor of $5 / 3$ for general graphs. This factor can be improved to $4 / 3$ when restricted to graphs with a perfect matching. 
Parameterized Complexity. Note that Lemma 19 actually shows that Edge InduCED KÖNIG SubGraPh is fixed-parameter tractable. To see this, suppose that $(G, k)$ is an instance of the problem; we are to decide whether $G$ has an edge induced König subgraph with at least $k$ edges. Note that if the parameter $k \leq 3 \mathrm{~m} / 5$ then we answer YES and use the approximation algorithm described in the previous subsection to obtain an edge induced König subgraph with at least $k$ edges. If $k>3 \mathrm{~m} / 5$ then we simply use a trivial $O^{*}\left(2^{m}\right)$ brute-force algorithm to decide the question. This FPT algorithm has time complexity $O^{*}\left(2^{5 k / 3}\right)$.

In this subsection, we give an $O^{*}\left(2^{k}\right)$ FPT algorithm for EDGE INDUCED KÖNIG SubGRAPH on connected graphs by using an exact algorithm for the optimization version of the problem. To this end, we describe an $O^{*}\left(2^{n}\right)$ algorithm for this problem using a simple structural result characterizing minimal König edge deletion sets of a graph.

Lemma 20. Let $G=(V, E)$ be a graph. If $E^{\prime}$ is a minimal König edge deletion set of $G$ then there exists $V^{\prime} \subseteq V$ such that $E\left(G\left[V^{\prime}\right]\right)=E^{\prime}$, that is, the edge set of the subgraph induced by $V^{\prime}$ is precisely $E^{\prime}$.

Proof. Let $E^{\prime}$ be a minimal König edge deletion set of $G$. Then $G^{\prime}=\left(V, E-E^{\prime}\right)$ is König. Then the vertex set of $G^{\prime}$ can be partitioned into $V_{1}$ and $V_{2}$ such that $V_{2}$ is a maximal independent set and there exists a matching saturating $V_{1}$ that lies across the cut $\left(V_{1}, V_{2}\right)$. Let $V^{\prime}=V_{2}$. Since $E^{\prime}$ is minimal, it is clear that $E\left(G\left[V_{2}\right]\right)=E^{\prime}$. This completes the proof.

Our exact algorithm for the optimization version of EDGE INDUCED KöNIG SUBGRAPH simply enumerates all possible subsets $V^{\prime} \subseteq V$, deletes all edges $E^{\prime}$ in $G\left[V^{\prime}\right]$ and checks whether $G-E^{\prime}$ is König. The algorithm returns an edge set $E^{\prime}=E\left(G\left[V^{\prime}\right]\right)$ of smallest size such that $G-E^{\prime}$ is König.

Lemma 21. Given an $n$-vertex graph $G=(V, E)$, the optimization version of the KÖNIG EDGE Deletion (and hence the optimization version of EDGE INDUCED KönIG SUbGRAPH) can be solved in time $O^{*}\left(2^{n}\right)$ and space polynomial in $n$.

Theorem 9. Edge Induced KöNig Subgraph can be solved in $O^{*}\left(2^{k}\right)$ time in connected undirected graphs.

Proof. Let $(G, k)$ be an instance of Edge Induced König Subgraph where $G$ is a graph with $m$ edges and $n$ vertices. A connected graph has a spanning tree which, being bipartite, is König. Since a tree has $n-1$ edges, if $k \leq n-1$ we answer YES; else $n \leq k+1$ and we use Lemma 21 to obtain an $O^{*}\left(2^{k}\right)$ time algorithm for Edge Induced König Subgraph.

\section{Conclusion and Open Problems}

In this paper, we introduced and studied vertex and edge versions of the KÖNIG SUBGRAPH problem from the points of view of parameterized complexity and approximation algorithms. Our results are summarized in Figure 4. We showed that KÖNIG VerTeX Deletion is FPT whereas Vertex Induced König Subgraph is $W[1]$-hard. The Edge Induced König Subgraph problem is FPT and we conjecture that KÖNIG Edge Deletion is $W[1]$-hard. Some obvious open problems are:

1. What is the parameterized complexity of the KÖNIG EDGE DeLETION problem?

2. Is there a better FPT algorithm for KÖNIG VERTEX DELETION perhaps without making use of the algorithm for Above Guarantee Vertex Cover?

3. Are there better approximation algorithms for all these problems? 


\begin{tabular}{|c|c|c|}
\hline Problem & $\begin{array}{l}\text { Parameterized } \\
\text { Complexity }\end{array}$ & Approximability \\
\hline $\begin{array}{l}\text { König Vertex Deletion } \\
\text { / Above Guarantee Ver- } \\
\text { Tex Cover }\end{array}$ & FPT. & $\begin{array}{l}O(\log n \log \log n) \text { approximation algorithm; NP-hard to approx- } \\
\text { imate to within } 1.7212 \text {; no constant-factor approximation algo- } \\
\text { rithm assuming UGC. }\end{array}$ \\
\hline König Edge Deletion & Open. & $\begin{array}{l}\text { NP-hard to approximate to within } 2.88 \text {; no constant-factor ap- } \\
\text { proximation algorithm assuming UGC. }\end{array}$ \\
\hline $\begin{array}{l}\text { VERTEX INDUCED KÖNIG } \\
\text { SUBGRAPH }\end{array}$ & $W[1]$-hard. & no factor- $O\left(n^{1-\epsilon}\right)$ approximation algorithm. \\
\hline $\begin{array}{l}\text { EDGE INDUCED KÖNIG SUB- } \\
\text { GRAPH }\end{array}$ & FPT. & $\begin{array}{l}\text { 5/3-approximation algorithm for general graphs; } 4 / 3- \\
\text { approximation algorithm for graphs with a perfect matching }\end{array}$ \\
\hline
\end{tabular}

Fig. 4. List of problems dealt with in this paper.

Acknowledgements. We thank Sundar Vishwanathan for pointing out Theorem 3.

\section{References}

1. A. Agarwal, M. Charikar, K. Makarychev and Y. Makarychev. $O(\sqrt{\log n})$ Approximation Algorithms for Min UnCut, Min-2CNF Deletion, and Directed Cut Problems. In Proceedings of the 37th ACM Symposium on Theory of Computing (STOC 2005), pp. 573-581, 2005.

2. J .M. Bourjolly and W. R. Pulleyblank. König-Egerváry Graphs, 2-Bicritical Graphs and Fractional Matchings. Discrete Applied Mathematics, Vol. 24, pp. 63-82, 1989.

3. M. Chlebik and J. Chlebikova. On Approximation Hardness of the Minimum 2-SAT Deletion Problem. Discrete Applied Mathematics, Vol. 155, Issue 2, pp. 172-179, 2007.

4. J. Chen And I. A. Kanj. On Approximating Minimum Vertex Cover for Graphs with Perfect Matching. Theoretical Computer Science, Vol. 337, pp. 305-318, 2005.

5. J. Chen, I. A. Kanj And G. XIA. Improved Parameterized Upper Bounds for Vertex Cover. In Proceedings of the 31st International Symposium on Mathematical Foundations of Computer Science (MFCS 2006), Springer LNCS, Vol. 4162, pp. 238-249, 2006.

6. R. W. Deming. Independence Numbers of Graphs - An Extension of the König-Egerváry Theorem. Discrete Mathematics, Vol. 27, pp. 23-33, 1979.

7. I. Dinur And S. SAfra. On the Hardness of Approximating Minimum Vertex Cover. Annals of Mathematics, Vol. 162, Issue 1, pp. 439-485, 2005.

8. R. Downey And M. R. Fellows. Parameterized Complexity. Springer-Verlag, New York, 1999.

9. J. Flum And M. Grohe. Parameterized Complexity Theory. Springer-Verlag, New York, 2006.

10. F. V. Fomin, F. Grandoni and D. Kratsch. Measure and Conquer: A Simple $O\left(2^{0.288 n}\right)$ Independent Set Algorithm. In Proceedings of the 17th Annual ACM-SIAM Symposium on Discrete Algorithms (SODA 2006), pp. 18-25, 2006.

11. M. R. Garey and D. S. Johnson. Computers and Intractability. W. H. Freeman and Company, New York, 1979.

12. F. Gavril. Testing for Equality Between Maximum Matching and Minimum Node Covering. Information Processing Letters, Vol. 6, Issue 6, pp. 199-202, 1977.

13. F. Gavril and M. Yannakakis. Edge Dominating Sets in Graphs. SIAM Journal of Applied Mathematics, Vol. 38, Issue 3, pp. 364-372, 1980.

14. J. Guo, J. Gramm, F. Hüffner, R. Niedermeier and S. Wernicke. Compression-Based Fixed-Parameter Algorithms for Feedback Vertex Set and Edge Bipartization. Journal of Computer and System Sciences, Vol. 72, Issue 8, pp. 1386-1396, 2006.

15. J. HÅstad. Clique is Hard to Approximate to Within $n^{1-\epsilon}$. Acta Mathematica, Vol. 182, pp. 105-142, 1999. 
16. S. Кнот. On the Power of Unique 2-Prover 1-Round Games. In Proceedings of the 34th Annual ACM Symposium on Theory of Computing (STOC 2002), pp. 767-775, 2002.

17. S. Khot and O. Regev. Vertex Cover Might be Hard to Approximate to Within $2-\epsilon$. Journal of Computer and System Sciences, Vol. 74, Issue 3, pp. 335-349, 2008.

18. P. N. Klein, S. A. Plotkin, S. Rao and E. Tardos. Approximation Algorithms for Steiner and Directed Multicuts. Journal of Algorithms, Vol. 22, Issue 2, pp. 241-269, 1997.

19. E. Korach, T. Nguyen and B. Peis. Subgraph Characterization of Red/Blue-Split Graphs and KönigEgerváry Graphs. In Proceedings of the 17th Annual ACM-SIAM Symposium on Discrete Algorithms (SODA 2006), pp. 842-850, 2006.

20. L. Lovász. Ear-Decompositions of Matching-Covered Graphs. Combinatorica, Vol. 3, pp. 105-118, 1983.

21. L. Lovász And M. D. Plummer. Matching Theory. North Holland, 1986.

22. M. Mahajan and V. Raman. Parameterizing Above Guaranteed Values: Max Sat and Max Cut. Journal of Algorithms, Vol. 31, Issue 2, pp. 335-354, 1999.

23. M. Mahajan, V. Raman and S. Sikdar. Parameterizing Above or Below Guaranteed Values. Journal of Computer and System Sciences, Vol. 75, Issue 2, pp. 137-153, 2009.

24. S. Mishra, V. Raman, S. Saurabh, S. Sikdar and C. R. Subramanian. The Complexity of Finding Subgraphs Whose Matching Number Equals the Vertex Cover Number. In Proceedings of the 18th International Symposium on Algorithms and Computation (ISAAC 2007), Springer LNCS, Vol. 4835, pp. 268-279, 2007.

25. H. Moser And D. M. Thilikos. Parameterized Complexity of Finding Regular Induced Subgraphs. In Proceedings of the 2nd Workshop on Algorithms and Complexity in Durham (ACiD 2006), pp. 107-118, 2006.

26. R. Motwani and P. Raghavan. Randomized Algorithms. Cambridge University Press, 1995.

27. R. Niedermeier. Invitation to Fixed-Parameter Algorithms. Oxford University Press, 2006.

28. V. Raman, S. Saurabh and S. Sikdar. Efficient Exact Algorithms Through Enumerating Maximal Independent Sets and Other Techniques. Theory of Computing Systems, Vol. 41, pp. 563-587, 2007.

29. I. Razgon And B. O'Sullivan. Almost 2-SAT is Fixed-Parameter Tractable. In Proceedings of the 35th International Colloquium on Automata, Languages and Programming (ICALP 2008), Springer LNCS, Vol. 5125, pp. 551-562. To appear in the Journal of Computer and System Sciences.

30. B. Reed, K. Smith And A. Vetta. Finding Odd Cycle Transversals. Operations Research Letters, Vol. 32, pp. 299-301, 2004.

31. H. Schröder, A. E. May, O. SÝkora And I. VrT'o. Approximation Algorithms for the Vertex Bipartization Problem. In Proceedings of the 24th Seminar on Current Trends in Theory and Practice of Informatics (SOFSEM 1997), Springer LNCS, Vol. 1338, pp. 547-554, 1997.

32. F. Sterboul. A Characterization of Graphs in which the Transversal Number Equals the Matching Number. Journal of Combinatorial Theory, Series B, Vol. 27, pp. 228-229, 1979.

33. C. R. Subramanian. Vertex Covers: Parameterizing Above the Requirement. IMSc Technical Report, February 2001

34. V. V. Vazirani. A Theory of Alternating Paths and Blossoms for Proving Correctness of the $O(\sqrt{V} E)$ General Graph Maximum Matching Algorithm. Combinatorica, Vol. 14, Issue 1, pp. 71-109, 1994.

35. S. Vishwanathan. On Hard Instances of Approximate Vertex Cover. ACM Transactions on Algorithms (TALG), Vol. 5, Issue 1, 2008.

36. D. Zuckerman. Linear Degree Extractors and the Inapproximability of Max Clique and Chromatic Number. In Proceedings of the 38th ACM Symposium on Theory of Computing (STOC 2006), pp. 681-690, 2006. 